\title{
An unfolding story on binding
}

\author{
IMAGE \\ UNAVAILABLE \\ FOR COPYRIGHT \\ REASONS
}

The type-Il restriction endonucleases of bacteria, which form part of a primitive defense system against entry of foreign DNA, have changed the face of molecular biology and biotechnology by providing clinically precise 'scissors' capable of cutting DNA in a highly predictable manner at specifically defined sites. At the same time, they present an intriguing biological problem in terms of understanding the basis of the rigid selectivity for their binding sites in DNA. The structure of Bam $\mathrm{HI}$ in complex with its recognition sequence 5'-GGATCC-3' (picture taken from M. Newman, T. Strzelecka, L. F. Dorner, 1. Schildkraut and A. Aggarwal, Science 269, 656-663; 1995) provides further insight into the recognition and cleavage mechanisms and demonstrates the first instance where DNA binding results in ordered secondary structure being transformed into disordered structure.

The upper image shows the free Bam HI dimer and the lower image shows the dimer in complex with the 12 base-pair recognition site (red: end on). One of the remarkable features of the new structure is the series of conformational changes that the protein undergoes (compare yellow segments in upper and lower panels) on binding to its target DNA sequence. The most surprising of these is the unfolding of the carboxy-terminal $\left(\alpha^{7}\right)$ helix (top panel: yellow

helices) of each subunit, which become extended and partially disordered (lower panel).

The helix in the right $(R)$ subunit unravels and grips the 'underside' of the DNA, binding in the minor groove (lower panel: bottom centre). The $\alpha^{7}$ helix in the L-subunit also unravels but cannot enter the minor groove because of the presence of the 'arm' from the R-subunit. Instead, the L-subunit arm folds back into the core of the protein, while following the path of the sugar-phosphate back bone of the DNA (bottom panel: lower left corner). The authors note that, in the free enzyme, the $\alpha^{7}$ helix is the most mobile and solvent accessible of the helices, and the energy barrier between the folded and unfolded states is likely to be low. Thus the new interactions that each arm gains in the protein-DNA complex ( $R$ with the minor groove and $L$ with the interior of the protein and DNA phophate back bone) may be sufficient to push the equilibrium over to the unfolded state.

Other changes on binding highlighted in the picture are: the ordering of a disordered loop (upper panel; centre top) to form a single turn of helix $\left(\alpha^{3 A}\right)$ which interacts with its symmetry related partner from the other subunit to form part of the dimer interface in the complex (lower panel; centre); local rearrangement of a second loop (upper panel: lower centre) which makes base-specific contacts with the outer and middle $\mathrm{G} \cdot \mathrm{C}$ base pairs of the recognition sequence in the major groove (lower panel: bracketing the DNA); and the rigid body motion of the dimers towards the DNA-binding cleft (upper panel; bottom centre) such that they 'grip' the DNA in the complex (lower panel: bottom centre). 